\title{
Segmental Aplasia of the Uterine Horn with Ipsilateral Renal Agenesis in a Cat
}

\author{
Jinhwa CHANG ${ }^{1)}$, Joo-hyun JUNG ${ }^{1)}$, Junghee YOON ${ }^{1)}$, Min-cheol CHOI ${ }^{1)}$, Jae Hak PARK ${ }^{2)}$, Kang-Moon SEO ${ }^{3)}$ and \\ Seong Mok JEONG ${ }^{4}$ * \\ ${ }^{1)}$ Departments of Veterinary Radiology, ${ }^{2)}$ Laboratory Animal Medicine and ${ }^{3)}$ Veterinary Ophthalmology/Surgery, College of Veterinary \\ Medicine, Seoul National University, San 56-1, Sillim-dong, Gwanak-gu, Seoul 151-742 and ${ }^{4)}$ Department of Veterinary Surgery, \\ College of Veterinary Medicine $\bullet$ Research Institute of Veterinary Medicine, Chungnam National University, 220, Gung-dong, Yuseong-gu, \\ Daejeon 305-764, Republic of Korea
}

(Received 1 December 2006/Accepted 19 February 2008)

ABSTRACT. A nine-month-old domestic short haired cat was admitted with the history of acute vomiting, depression and shivering. Abdominal ultrasonography revealed minimum enlargement of the right uterine horn filled with anechoic fluid. On excretory urography, functionally and anatomically normal, enlarged left kidney was found, but right kidney was absent. It was preliminary diagnosed as hydrometra with right renal agenesis. Aiming at the correction of hydrometra, we performed ovariohysterectomy. During spaying, we found a missing segment of distal part of the right uterine horn and absence of ipsilateral kidney and ureter. Compressed uterine structure and segmental aplasia of right uterine horn were found in histopathological investigation. Taken together, it was diagnosed as a segmental aplasia of uterine horn with ipsilateral renal agenesis.

KEY WORDS: feline, renal agenesis, uterine segmental aplasia.

A nine-month-old, intact female, domestic short haired cat was admitted with the history of acute vomiting, depression and shivering for three days. The rise of temperature $\left(40^{\circ} \mathrm{C}\right)$ was recorded, and there was azotemia (BUN: 74.0 $\mathrm{mg} / \mathrm{d} l$, creatinine: $4.5 \mathrm{mg} / \mathrm{d} l$ ) on the routine blood screening test. Abdominal radiography showed absence of the right kidney, while the left one was enlarged $(3.2 \times$ length of the 2 nd lumbar vertebra). Incidentally, there was unilateral sacralization of the 7 th lumbar vertebra with articulation between right transverse process and the ilium at same side. Abdominal ultrasonography demonstrated minimum enlargement of the right uterine horn (12.5 $\mathrm{mm}$ in diameter) filled with anechoic fluid. Excretory urography revealed the normal shape and opacification pattern of the left kidney meaning the normal physiological activities, however, the right kidney was absent (Fig. 1). In spite of unknown causes of azotemia, this patient was recovered through 2 days supportive treatment including fluid therapy and diuretics, and then received ovariohysterectomy under general anesthesia to prevent further deterioration of the uterine horn. Surprisingly, there were no the distal part of the right uterine horn, the right kidney and the right ureter during surgery (Fig. 2A). The remnant of the right uterine horn was tortuous and dilated with fluid, and was not connected to the body of the uterus. The left uterine horn was intact and there was a small notch as vestige at the body of uterus (Fig. 2B). Gross and histopathologic examination revealed that both ovaries, fallopian tubes and the left uterine horn were normal (Fig. 3A-C). There were many follicles showing various stages and the majority of the follicles underwent degeneration in

\footnotetext{
* Correspondence to: Jeong, S. M., Department of Veterinary Surgery, College of Veterinary Medicine $\cdot$ Research Institute of Veterinary Medicine, Chungnam National University, 220, Gung-dong, Yuseong-gu, Daejeon 305-764, Republic of Korea. e-mail: jsmok@cnu.ac.kr
}

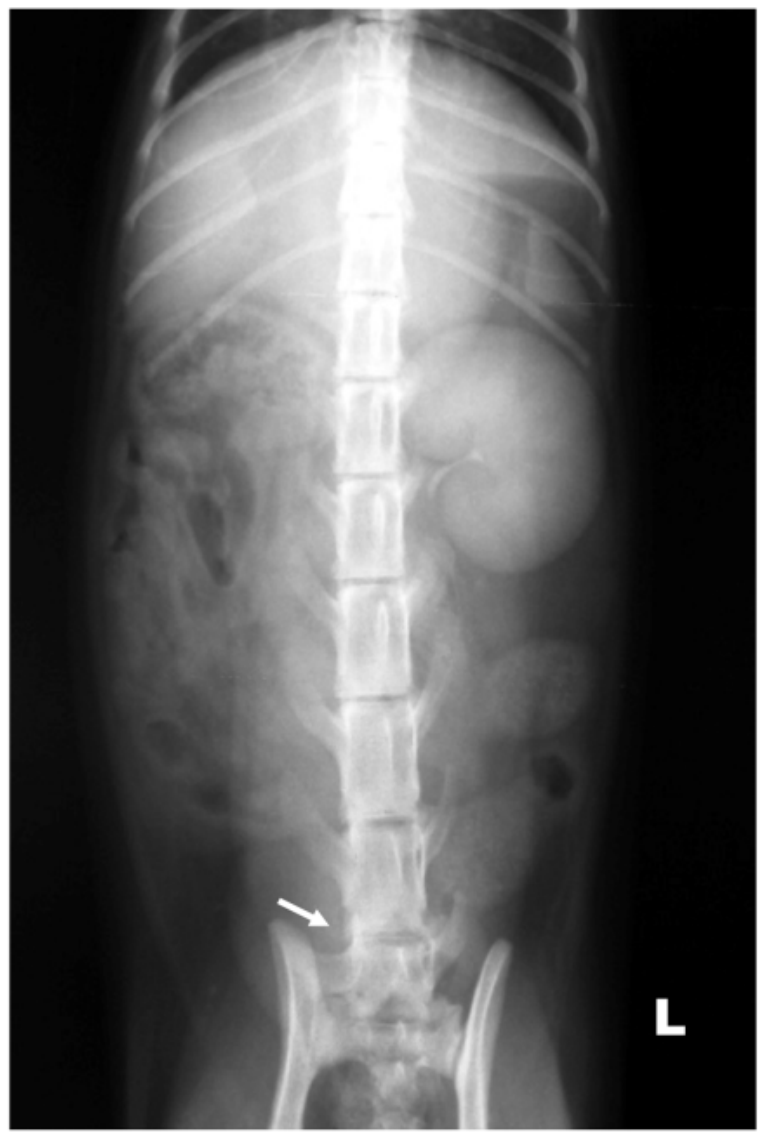

Fig. 1. Ventrodorsal radiogram of the cat. The right kidney is not visualized and the left kidney is enlarged but appears anatomically and functionally normal on the excretory urogram. There is unilateral sacralization of the 7th lumbar vertebra with articulation between right transverse process and the ilium at same side (arrow). 


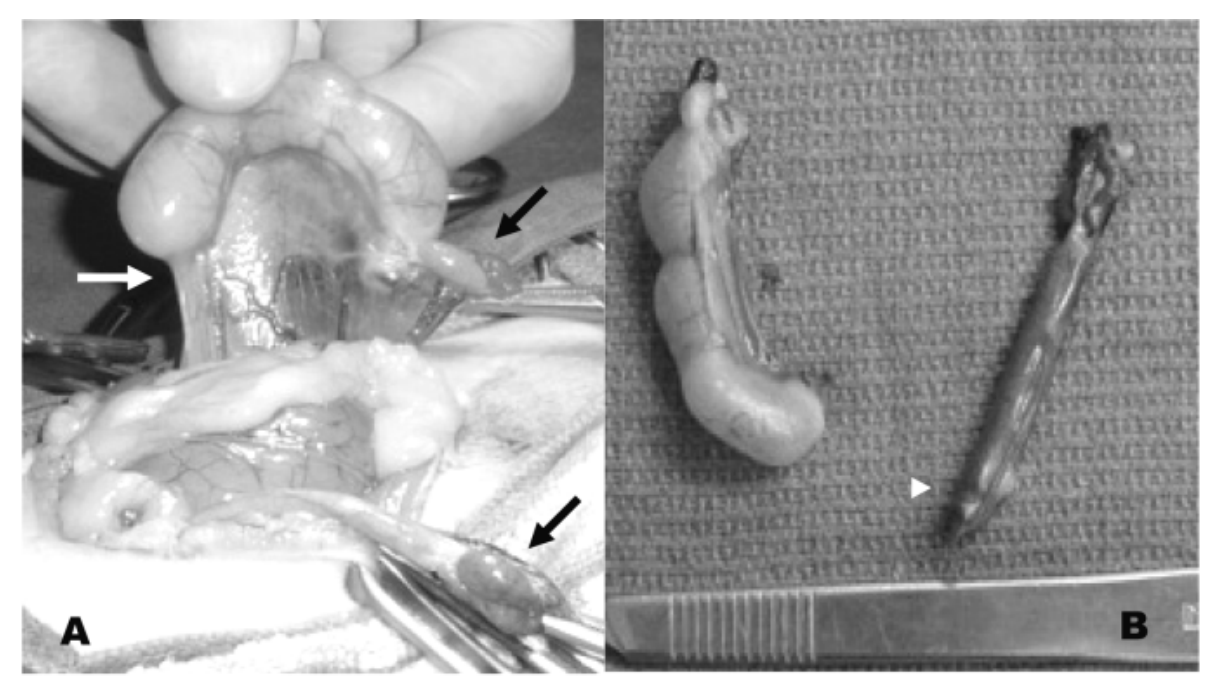

Fig. 2. A; Intraoperative photograph of the cat. The missing segment of distal part of the right uterine horn is not connected to the uterine body (white arrow). Blind ended right uterine horn is dilated (diameter, $12.5 \mathrm{~mm}$ ) and tortuous. Both ovaries reveal normal appearance (black arrows). B; The left uterine horn is intact and there is a small notch at the uterine body (arrow head).
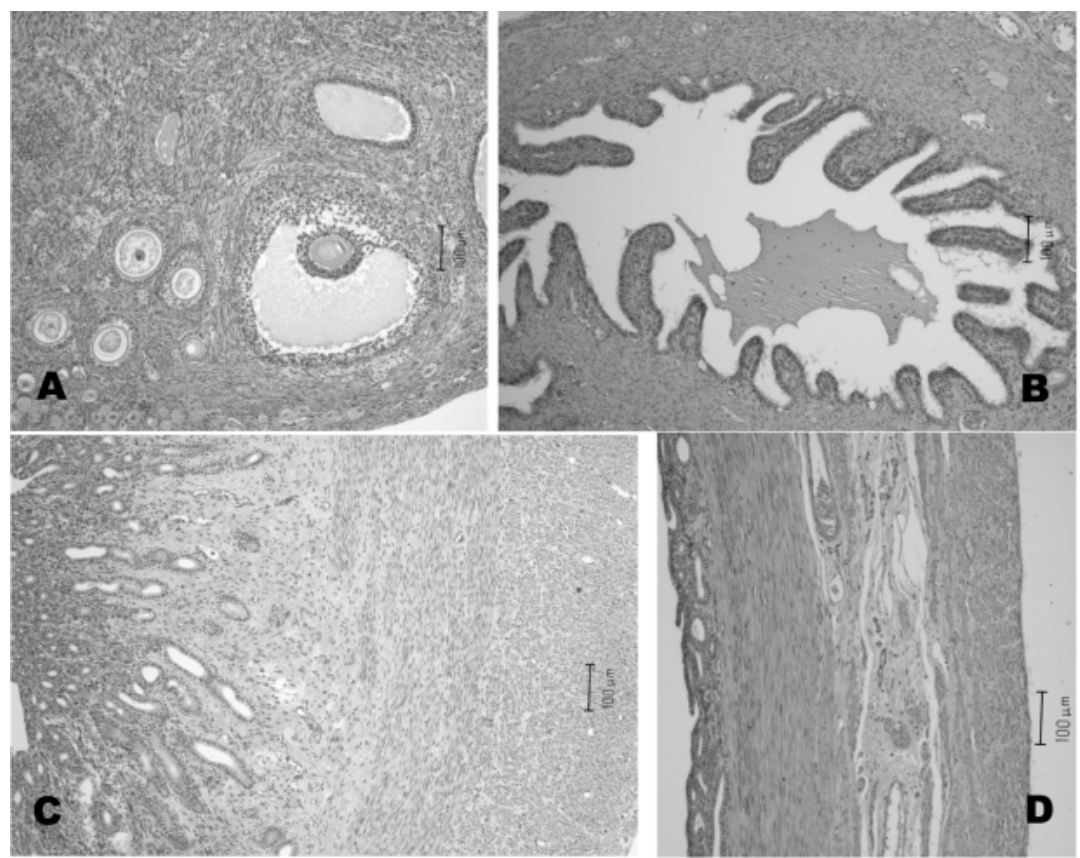

Fig. 3. Histological findings of the cat with uterine segmental aplasia (H\&E stain). A; There are many follicles showing with degeneration and no corpus luteum in the ovaries. B; Both fallopian tubes are normal. C; The left uterus shows normal appearance with thick endometrium and muscular layers. D; On the dilated right uterine horn, the number of endometrial glands are markedly reduced and most of them are compressed. The muscular and vascular layers became slightly thin.

the ovaries. No corpus luteum was seen in both ovaries (Fig. 3A). The right uterine horn showed sterile hydrometra with compressed uterine structure and distal segmental aplasia (Fig. 2A, Fig. 3D).

Segmental aplasia of genital tract means missing seg- ments of the uterus, cervix, and/or vagina of the genital tract $[1,5,8]$. These rare disorders of the reproductive tract are seen most frequently in the cow and sow, occasionally in the cat as well $[3,7]$. The majority of uterine congenital anomalies involve the uterine horns [5]. Segmental aplasia of a 
portion of a uterine horn results from segmental defects in paramesonephric, or Müllerian duct development [2, 3, 5, 9]. Congenital developmental abnormalities such as bilateral agenesis, unilateral agenesis including uterus unicornis or hemiuterus, and segmental aplasia is mainly due to malformation (agenesis, hypoplasia) of Müllerian duct [2, 3].

Embryologically, Müllerian ducts are differentiated into oviducts, uterus, uterine tube and vagina $[2,6]$. Absence or segmental aplasia of one or both uterine tubes, one uterine horn, the uterine body, or vagina is due to abnormal differentiation of Müllerian duct development [7]. These congenital defects are associated with hermaphroditic conditions or with ipsilateral renal agenesis because of the close developmental association of the urinary and genital systems [7]. In human with Müllerian duct anomalies, a range of coexisting renal anomalies including renal agenesis, renal ectopia, horseshoe kidney or pelvic kidney can be seen [1]. The Müllerian anomaly with secondary ipsilateral renal agenesis is almost twice more common on the right than the left side of body [10]. Similar defects with this trend in the urogenital system of feline have also been reported [7-9]. The coexisting association between urinary and genital malformation has long been recognized, however, they have not been properly studied.

In case of unilateral renal agenesis, there was commonly compensatory hypertrophy of the contralateral kidney $[4,9]$. A suitable procedure should be conducted in advance of urinary system investigation in those patients suspected to have genital malformation. In general, excretory urography has been practiced to confirm anatomic abnormalities, and the functional status of the kidney.

This patient suffered from segmental aplasia had anechoic fluid in the affected uterine horn. It was due to accumulation of secretes in the blind-ended segments. There might be several potential causes; hormonal influ- ences after estrus cycle, chronic cystic endometrial hyperplasia, structural malformation [2,7]. Segmental aplasia of the uterine horn is a rare developmental disorder that is difficult to trace without invasive investigation, and too hard to predict obvious genital clinical signs. Unless it is bilateral, fertility is not necessarily impaired. However, the exact prevalence and actual clinical profiles are not reported due to its peculiar characteristics. It is commonly found by exploratory laparotomy or the incidental finding in ovariohysterectomy. It is necessary to perform histopathologic examination to assess the type of uterine anomaly, and additional attention should be drawn towards the concomitant investigation on urinary system.

\section{REFERENCES}

1. Acin, P., Acin, M. and Snchez-Ferrer, M. 2004. Hum. Reprod. 19: $2377-2384$.

2. Gee, B. R., Pharr, J. W. and Furneaux, R. W. 1977. Can. Vet. J. 18: $281-286$.

3. Ginther, O. J. 1965. J. Am. Vet. Med. Assoc. 146: 133-137.

4. Greco, D. S. 2001. Vet. Clin. North Am. Small Anim. Pract. 31: 393-399.

5. Jones, T. C., Hunt, R. D. and King, N. W. 1997. pp. 1149 1221. In: Veterinary Pathology. 6th ed. (Jones, T. C., Hunt, R. D. and King, N. W. eds.), Lippincott Williams \& Wilkins, Philadelphia.

6. Mack, C. O. and McGlothlin, J. H. 1949. Anat. Rec. 105: 445450.

7. Marcella, K. L., Ramirez, M. and Hammerslag, K. L. 1985. J. Am. Vet. Med. Assoc. 186: 179-181.

8. Memon, M. A. and Schelling, S. H. 1992. Vet. Rec. 131: 266267.

9. Robinson, G. W. 1965. J. Am. Vet. Med. Assoc. 147: 516-518.

10. Vercellini, P., Daguati, R., Somegliana, E., Vigano, P., Lanzani, A. and Fedele, L. 2007. Fertil. Steril. 87: 719-724. 